\title{
Preferred rates of repetitive tapping and categorical time production
}

\author{
CHARLES E. COLLYER \\ University of Rhode Island, Kingston, Rhode Island \\ and \\ HILARY A. BROADBENT and RUSSELL M. CHURCH \\ Brown University, Providence, Rhode Island
}

\begin{abstract}
In a constrained finger-tapping task, in which a subject attempts to match the rate of tapping responses to the rate of a pacer stimulus, interresponse interval (IRI) was a nonlinear function of interstimulus interval (ISI), in agreement with the results of Collyer, Broadbent, and Church (1992). In an unconstrained task, the subjects were not given an ISI to match, but were instructed to tap at their preferred rate, one that seemed not too fast or too slow for comfortable production. The distribution of preferred IRIs was bimodal rather than unimodal, with modes at 272 and $450 \mathrm{msec}$. Preferred IRIs also tended to become shorter over successive sessions. Time intervals that were preferred in the unconstrained task tended to be intervals that were overproduced (IRI > ISI) when they were used as ISIs in the constrained task. A multiple-oscillator model of timing developed by Church and Broadbent (1990) was used to simulate the two tasks. The nonlinearity in constrained tapping, termed the oscillator signature, and the bimodal distribution in unconstrained tapping were both exhibited by the model. The nature of the experimental results and the success of the simulation in capturing them both provide further support for a multipleoscillator view of timing.
\end{abstract}

Collyer, Broadbent, and Church (1992) recently reported a finding in the area of motor control termed categorical time production (CTP). When subjects were required to tap their finger at a specified rate, some rates were consistently reproduced too slowly, and some were reproduced too quickly. We termed the pattern of rate biases the oscillator signature. The pattern of relatively slow and relatively fast rates supported a categorical timing hypothesis, in which time production is controlled by a set of oscillatory processes, each with a natural rate of its own.

If the hypothesized oscillatory processes are part of a general timing mechanism, we would expect their influence to be observed across a variety of timing tasks. Furthermore, we would expect a subject's performance in one task to be related to his or her performance in another, by virtue of the common involvement of these processes. In the present study, we tested a group of subjects on two timing tasks, the constrained-rate tapping task, in which

This work was supported by National Science Foundation Grants BNS9109638 to the University of Rhode Island and BNS9110158 to Brown University, and by Grant NSS2-S07-RR07086-14 to the University of Rhode Island from the National Center for Research Resources. The authors thank York Maksik for superb technical assistance. Some of the data were presented at the meetings of the New England Sequencing and Timing Society in January 1992 and February 1993. Correspondence should be addressed to C. E. Collyer at the Department of Psychology, University of Rhode Island, Kingston, RI 02881. the evidence for CTP was originally demonstrated, and an unconstrained-rate (preferred-rate) task. We sought to determine the relationship between subjects' preferred rate of tapping and their oscillator signature in the constrained task.

\section{The Oscillator Signature Resembles a Rounding-Error Function}

The pattern of errors in time production discovered by Collyer et al. (1992) resembles a rounding-error function. Consider a high-precision quantity $H$ rounded to a lowerprecision quantity $L$. The possible lower-precision values or categories are often, but not necessarily, the integers. Rounding error is defined as the difference $D=L-H$. Different rounding rules are possible, such as rounding up or down to the next candidate value of $L$, or rounding to the candidate value of $L$ nearest to $H$. If the lower precision values are spaced at regular intervals on the original $H$ scale-for example, as integers are spaced on the scale of real numbers-the function relating $D$ to $H$ will be periodic. Figure 1 gives examples of rounding for the cases of rounding up to the next higher integer (top panels), rounding to the nearest integer (middle panels), and rounding down to the next lower integer (bottom panels).

The middle panels of Figure 1 show the familiar case of rounding to the nearest integer. The heavy line in the left panel illustrates that $L$ is a step function of $H$, with the middle of each step falling on the identity function. The right panel shows residuals, $R$, from a linear fit to the data of the left panel. (In this case only, $R=D$.) 


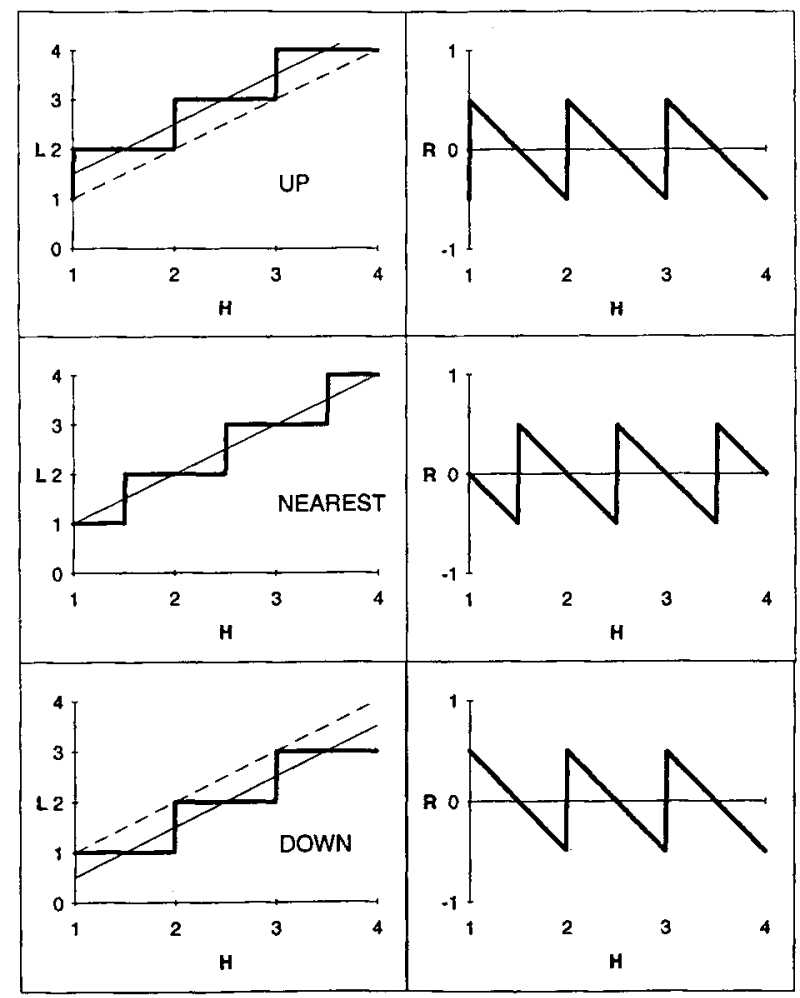

Figure 1. Rounding error provides an analogy to categorical time production. Three cases of rounding real numbers to integers are illustrated here. Panels on the left show the mapping of higher precision values, $H$, to lower precision values, $L$, for the cases of rounding up (top), rounding to the nearest integer (middle), and rounding down (bottom).The identity function is indicated by a dashed line. The best linear fit to the rounded values is indicated by a solid straight line. The panels on the right show residuals from the bestfitting straight line for these three cases. These residual plots are analogous to oscillator signatures and reflect the periodic spacing of integers on the real number line. Integers are associated with negative-going zero crossings of the residual function for the middie case, because values are rounded symmetrically toward the middle of a rounding category. In the other two cases, the integers are at the positive-going transitions. Thus, the interpretation of the residual function in terms of the location of $L$ values depends on which type of rounding underlies the function.

Negative-going zero crossings of the residuals occur at the value of $H$ equal to the $L$ of each rounding category (e.g., $H=2$ ). Transitions from negative to positive residuals occur at category boundaries (e.g., $H=2.5$ ).

The top panels of Figure 1 show rounding up to the next higher integer. The left panel shows that, in this case, all but the right edge of each step falls above the identity function. A linear fit to the rounded values would bisect the steps, but not at values of $H$ equal to values of $L$. The right panel shows that the integer values of $H$ are at positive-going transitions. Similarly, the bottom panels of Figure 1 show the case of rounding down. Again, the integer values are at positive-going rather than negativegoing transitions of the residual function.

The precision of psychological time intervals is limited relative to physical intervals, in a way analogous to the limited precision of rounded numbers (Collyer et al., 1992). In our earlier report, we conjectured that negativegoing zero crossings could be used to estimate the natural periods of the timing system responsible for the oscillator signature. This would be so if the categorization of time intervals were analogous to the symmetrical rounding shown in the middle panels of Figure 1. However, it is clear that natural periods could be associated with other parts of the signature, just as the integers can be associated with different parts of the residual functions of Figure 1. In this paper, we consider evidence and theory relevant to the interpretation of oscillator signatures in terms of natural periods.

The residual functions in Figure 1 have the appearance of a sawtooth wave with abrupt transitions from negative to positive bias and more gradual transitions from positive to negative bias. In contrast, oscillator signatures have gradual positive-going as well as negative-going zero crossings. How could a rounding process give rise to gradual positive-going zero crossings? One possibility is that the criterion determining whether rounding will be up or down, is variable. For example, the criterion for rounding up to 3 is usually 2.5 . If this criterion fluctuated randomly among the values $2.4,2.5$, and 2.6 , the average rounding errors associated with values of $H$ in this part of the range would exhibit a more gradual transition than in the case of a constant criterion. Another possibility is that neighboring values of $L$ exert a weighted joint influence on the final rounded value. Thus, with some modifications, a rounding analogy can accommodate gradual transitions in the residual function.

Collyer et al. (1992) measured the zero crossings of the oscillator signatures of 5 subjects. On average, there were negative-going crossings at time intervals of 250 and $513 \mathrm{msec}$, and positive-going crossings at 413 and 748 msec. Did these zero crossings signify natural periods, category boundaries, or another feature of a timing mechanism? One way to address this question is to examine how preferred rates of tapping are distributed relative to such features of the oscillator signature.

\section{Multiple Oscillatory Processes and Units of Time}

To clarify our conception of oscillatory processes in timing, let us consider the ordinary measurement of time in hours and days. A day is a unit of duration made up of smaller units, hours, which serve to specify within-day times. Any of 168 distinct hours could be specified by two oscillators, one with a series of 7 distinguishable states representing days of the week, and another with a series of 24 distinguishable states representing hours within a day. Calendars and clocks are concrete models of these series, and they are both oscillatory in that the series of states repeat themselves.

Continuing the hour-day example, various intervals of time can be specified to within an hour with the two oscillators. We could refer to the changing states of the oscillators in asking someone to meet us 2 days and 5 hours from now. The two oscillators also provide a way of discerning different rates of change. For example, an event 
that recurs at intervals of over an hour would display a changing phase lag relative to the hour oscillator (later and later with each cycle), opposite to that of an event recurring at intervals of under an hour (earlier and earlier with each cycle). Conventional time units thus provide a familiar example of a system in which time is measured with reference to oscillators. Such a system is capable of all the familiar uses to which time measurement is put, such as specifying particular times, measuring durations, and representing rates of change.

We suggest that psychological time is regulated by a system of oscillators whose functions are analogous to those of such time references as calendars and clocks. Our focus is on relatively short time intervals used to define rates of repetitive finger-tapping. Finger-tapping provides a number of convenient tasks for studying a person's ability to perceive and control the timing of voluntary actions.

\section{Constrained and Unconstrained Tapping}

In a constrained tapping task, trials are typically divided into two phases, synchronization and continuation (Wing, 1980; Wing \& Kristofferson, 1973). During the first phase, synchronization, the subject attempts to synchronize finger taps with auditory pulses presented over headphones at a constant interstimulus interval (ISI). During the second phase, continuation, the subject attempts to maintain the same rate of tapping as in synchronization, but without the auditory pulses. Collyer et al. (1992) found the oscillator signature in the continuation phase of constrained tapping trials. It appeared that there were time intervals toward which both shorter and longer intervals were "rounded," a finding consistent with the idea of oscillators with natural periods. There appeared to be individual differences in the duration of these periods. Finally, a subject's performance in these respects was invariant across the biomechanically different conditions of finger and wrist flexion.

In unconstrained tapping, trials are, in a sense, all continuation. The subject is asked to tap at a rate that feels subjectively natural-not too fast or too slow. In an exploration of these "preferred rates" of tapping (Collyer, Broadbent, \& Church, 1990), we found that subjects exhibited individual differences, but the rates were reliable within subjects over two sessions. Preferred rates under finger and wrist flexion did not differ.

The present experiment involved both constrained and unconstrained tapping and was carried out over several sessions. The two principal aims were to replicate the oscillator signature in constrained tapping and to examine the relationship between the oscillator signature and preferred rates in unconstrained tapping.

\section{METHOD}

\section{Subjects}

The subjects were 4 men and 12 women, ranging in age from 19 to 41 . They were recruited at the University of Rhode Island. All were predominantly right-handed. Four graduate students and 2 undergraduate students in the group were familiar with the gen- eral purposes of the research but not with specific hypotheses at the time they served as subjects. The subjects were paid $\$ 40$ for a series of eight sessions lasting less than $1 \mathrm{~h}$ each. The eight sessions were usually run on different days.

\section{Apparatus}

The experiment was controlled by an IBM PS/2 Model 30-286 computer with AT internal architecture. Timing of auditory stimuli was controlled, and timing of responses measured, by a Metrabyte CTM05 I/O board. Auditory stimuli ( $50-\mathrm{msec}, 1-\mathrm{kHz}$ tones) were generated by a MED Associates signal generator (ANL-916). The subjects heard the tones over headphones (Sony Walkman type).

The system used to detect the subjects' tapping responses consisted of an infrared emitter and detector mounted $4 \mathrm{~cm}$ apart on the aluminum cover of a small plastic box (dimensions $8.3 \times 5.5$ $\times 3.0 \mathrm{~cm}$ ). The emitter was an infrared/near-infrared high-output light-emitting diode (SYIR53L, Radio Shack Part 276-145) pulsed on and off at a rate of $22.5 \mathrm{KHz}$ by an oscillator circuit contained in the box. The detector was a phototransistor (SY32PT, Radio Shack Part 276-145) connected to a missing pulse detector circuit. This circuit sent a .1-msec pulse to an interrupt line of the CTM05 board when the detector failed to receive one of the pulses generated by the emitter. This pulsed emitter-detector arrangement was used to cut down on errors created by background infrared radiation (see Maksik, 1991). A small wooden block (about $1 \mathrm{~cm} \mathrm{high)}$ was placed halfway between the emitter and detector to provide a consistent target for the subject to tap on. The infrared beam extended from .1 to $1 \mathrm{~mm}$ above the target, and the detector required $80 \%$ occlusion of the beam to record a response. Testing indicated that a "finger down" event was recorded when the subject's finger was about $.22 \mathrm{~mm}$ above the target; a "finger up" event was recorded when the finger was at about the same distance. The total circuit response time to $80 \%$ occlusion was less than $.05 \mathrm{msec}$. Occasionally, a tap missing the beam would result in the recording of an interresponse interval (IRI) of about twice the normal duration. We minimized the influence of outliers in the data by using medians instead of means and semiinterquartile ranges instead of standard deviations.

\section{Design and Procedure}

A constrained tapping trial consisted of 50 synchronization taps and 50 continuation taps. During the synchronization phase of a trial, a train of 50-msec auditory tones (pacer sounds) at some constant ISI was heard over the headphones. The subject's task was to tap in synchrony with these sounds. After 50 synchronization taps, there were no further pacer sounds. The subject's task was to continue tapping at the same rate. The trial ended after 50 of these taps in the continuation phase without pacer sounds. The end of a trial was signaled by a distinctive sound (two short tones). There were 28 values of ISI, ranging from 200 to $875 \mathrm{msec}$ in steps of 25 msec. Each subject had a different random permutation of the 28 values. A block of five trials was run at each ISI. Four blocks were run in each data session, and there were seven sessions.

An unconstrained tapping trial consisted of a single auditory pulse to signal the start of the trial, followed by 100 taps. The subjects were instructed to tap at a steady rate that seemed comfortable to them-not too fast or too slow-for continued production. Sessions began and ended with sets of three unconstrained tapping trials. Thus, the structure of one session was the following: three unconstrained tapping trials, five constrained tapping trials at each of four ISIs, blocked, and three more unconstrained tapping trials.

The subjects were tested individually in a quiet room while seated at a table and wearing headphones. Tapping was done with the index finger of the right hand. The subjects held the response box with the thumb and remaining fingers of the right hand while tapping. The box rested on a typewriter pad to prevent extraneous movement. 
One subject completed two replications of the constrained tapping procedure to assess the within-subject reliability of her oscillator signature.

\section{Comments on Analyses}

Percent Residual Bias (PRB). Following Collyer et al. (1992), we partitioned each subject's total bias at a particular constrained rate of tapping (IRI-ISI) into a component linearly related to ISI and a residual component. The residual bias was expressed as a percentage of the linear prediction at each ISI. In the earlier study, we found that residual bias displayed wavelike nonlinear variation as a function of ISI, as well as random error. The percent transformation provided an adjustment for the roughly scalar increase in residual variability with increasing ISI.

Smoothing of oscillator signatures. Often it is desirable to reduce the noise in a data series in order to reveal the most important features. When the underlying function form of the series is known, curve-fitting can serve this purpose. When the form is unknown, a statistical filter or smoothing algorithm may yield satisfactory results. An oscillator signature is a type of data series for which simplification would be helpful but for which an exact function form is not known.

Tukey (1977) and Velleman and Hoaglin (1981) have described several exploratory procedures for smoothing data series. These procedures typically involve more than one computational operation on the series. The purpose of the early operations is to remove outliers that may not belong to the main pattern; the later steps use weighted averages of neighboring points to reduce local noise in the data. Steps for smoothing end points of a series and for recovering information from residuals have also been described by these authors.

One compound procedure recommended by Velleman and Hoaglin (1981), and which we have adopted to simplify oscillator signatures, is termed the $4253 \mathrm{H}$, twice smoother. The name outlines the sequence of computational operations: on the first pass, the signature is recoded using running medians of four values; on the second pass, these medians are replaced by the means of adjacent pairs, and so on. A full description of this procedure can be found in Velleman and Hoaglin (1981).

\section{RESULTS}

Results will be reported for unconstrained tapping, constrained tapping, and the relationship between the two tasks.

\section{Unconstrained (Preferred Rate) Tapping Performance}

Each preferred rate trial was summarized using the median and semiinterquartile range of the 99 IRIs defined by 100 taps. The overall mean of trial median IRIs was $408.3 \mathrm{msec}$, and the mean of trial semiinterquartile ranges was $13.3 \mathrm{msec}$, or $3.3 \%$ of the typical preferred IRI.

Sessions began and ended with a group of three preferred rate trials. Preferred IRIs from trials at the start and at the end of a session did not differ significantly $[t(15)=0.42]$. The Pearson correlation between the start and end IRIs was high $[r(14)=.91, p<.01]$. Thus, the subjects' preferred IRIs exhibited characteristic individual values that were relatively unaffected by the constrained tapping task that intervened between the start and end series.

Preferred IRIs exhibited a small degree of slowing, on average, within trials. A measure of within-trial drift was the median difference between adjacent IRIs. The mean of these medians was taken for each group of three trials (one group at the beginning of a session and one at the end). There were 112 such means (16 subjects $\times 7$ sessions) for beginning trials and 111 for end trials, with one missing value. The overall mean drift was $0.165 \mathrm{msec}$ per tap. This value is significantly different from zero $[t(222)=1.97, p<.05]$ and amounts to an average increase in IRI of $16.3 \mathrm{msec}$, or $4 \%$, over the course of a 99 -interval preferred rate trial. Because this within-trial drift was small, we continued to summarized tapping rates with the median within-trial IRI.

Preferred IRI tended to drop with repeated measurement in two ways, which are illustrated in Figure 2. First, IRI decreased within a group of three preferred rate trials. The three trials at the beginning of a session had average (mean over subjects of the mean of trial medians) preferred IRIs of 433, 405, and $399 \mathrm{msec}$, which differed significantly $[F(2,30)=9.6, p<.01]$. Follow-up tests showed that the first trial differed from the second $[t(15)=3.3, p<.01]$ and from the third $[t(15)=4.1$, $p<.01]$ but that the second and third trials did not differ from each other $[t(15)=0.8]$. The three trials at the end of a session had average preferred IR Is of 420,402 , and $400 \mathrm{msec}$, which differed significantly $[F(2,30)=12.3$, $p<.01]$. Follow-up tests showed that the first trial differed from the second $[t(15)=4.1, p<.01]$ and from the third $[t(15)=4.5, p<.01]$ but that the second and third trials did not differ from each other $[t(15)=0.3]$.

Second, there was a tendency for preferred IRIs to decrease over sessions. Ten of the 16 subjects exhibited significant speedup as measured by linear regression analyses of each series of 42 trial median IRIs. No subjects exhibited significant slowing over sessions in the regression analysis. Figure 2 shows median preferred rate as a function of successive trials.

The regression analysis of the decrease in preferred rate assumed an underlying linear change over trials. However, the theoretical direction of our earlier work (Collyer et al., 1992) would suggest that discontinuous changes

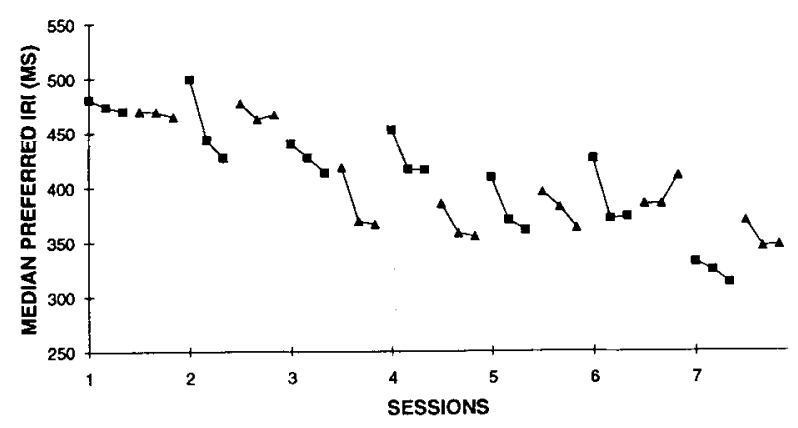

Figure 2. Decrease of preferred interresponse interval over sessions. Each data point is the mean of 16 trial medians ( 1 for each subject). Data points connected by lines belong to the same set of three consecutive trials. Rectangles and triangles denote trials at the beginning and at the end of a session, respectively. 


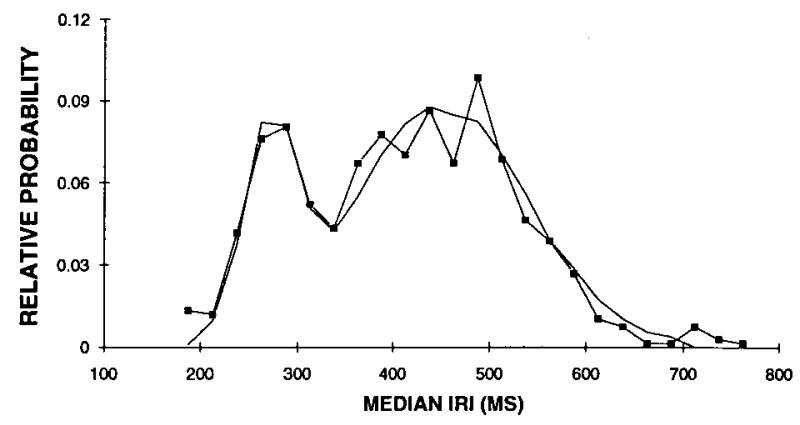

Figure 3. Distribution of 670 trial median preferred interresponse intervals, using 25 -msec bins. The bimodal curve is the mixture of two normal distributions. We suggest that the two component distributions arise from different temporal categories.

also be considered, such as a shift from one relatively discrete preferred IRI to another. One type of support for discontinuity was the shape of the distribution of trial median preferred IRIs, shown in Figure 3. This distribution was very well described by a bimodal mixture of two normal distributions, with means of 272 and $450 \mathrm{msec}$, and standard deviations of 25 and $90 \mathrm{msec}$, respectively. The mixture shown in Figure 3 weighted the shorter IRI component distribution 0.2 and the longer IRI component 0.8 .

This way of conceptualizing the set of trial preferred rates led to a three-parameter step-function model of the decrease over sessions. The three parameters were the IRI of the first step, the IRI of the second step, and the trial of transition from the first step to the second. The model was applied to each subject's series of 42 trial median IRIs, and the best-fitting combination of the three parameters (by a least-squares criterion) was found by iterative search. Parameter estimates for both the linear regression and the step-function analyses are shown in Table 1.

The average proportion of variance accounted for by the step model was almost double that of the linear model. It should be noted that a better fit by the three-parameter step model was not guaranteed in this analysis, although such guarantees usually apply in comparisons of strong and weak models (Collyer, 1985). The step model would have accounted for about .75 of the variance in a perfectly linear set of data. The fit of the step model to the 42 trial IRIs was significantly better than that of the linear model for 6 subjects ( $t$ tests with $40 d f, p<.01$ ). The fit of the linear model was significantly better for 1 subject.

Can the change in preferred rate be viewed as a shift from one natural period to another? In the range of intervals where preferred IRIs are found, the oscillator signatures of Collyer et al. (1992) would suggest that the difference between natural periods is about $263 \mathrm{msec}$, the difference between negative-going zero crossings, or $335 \mathrm{msec}$, the difference between positive-going zero crossings. Visual inspection of Figure 2 suggests that the average change in preferred rate over sessions is about $150 \mathrm{msec}$. Individual slope estimates from the linear model gave changes over the $\mathbf{4 2}$ trials ranging from an increase of $134 \mathrm{msec}$ to a decrease of $370 \mathrm{msec}$, with the mean change being a decrease of $105 \mathrm{msec}$. The step model gave step pairs that differed by an average of $122 \mathrm{msec}$, with a range of from 31 to $285 \mathrm{msec}$. Under either model, 2 or 3 subjects could be described as changing their preferred rate enough to suggest a shift from one natural period to another. However, most subjects exhibited smaller changes.

Table 1

Linear and Step Model Analyses of Change in Preferred Rate

\begin{tabular}{|c|c|c|c|c|c|c|c|c|}
\hline \multirow[b]{2}{*}{ Subject } & \multirow[b]{2}{*}{$M$} & \multicolumn{3}{|c|}{ Linear Regression Fit } & \multicolumn{4}{|c|}{ Step Function Fit } \\
\hline & & Slope & Intercept & $\operatorname{VAF}\left(r^{2}\right)$ & Step 1 & Step 2 & Break & VAF \\
\hline 1 & 358.6 & -5.8 & 483.5 & $.447^{*}$ & 458.1 & 303.3 & 15 & $.496^{*}$ \\
\hline 2 & 252.0 & -2.0 & 294.4 & $.269 *$ & 270.2 & 206.3 & 30 & $.392 *$ \\
\hline 3 & 387.5 & -1.0 & 408.5 & .029 & 405.0 & 282.5 & 36 & $.376^{*}$ \\
\hline 4 & 469.3 & 1.0 & 446.8 & .019 & 442.1 & 518.2 & 27 & $.159^{*}$ \\
\hline 5 & 380.7 & -8.8 & 569.7 & $.654^{*}$ & 79.7 & 281.8 & 21 & $.565^{*}$ \\
\hline 6 & 444.5 & -2.1 & 488.9 & $.225 *$ & 89.1 & 417.1 & 16 & $.440^{*}$ \\
\hline 7 & 461.6 & -2.5 & & .31 & & 441.8 & 10 & $.423^{*}$ \\
\hline 8 & 477.2 & -2.8 & 538.3 & $.165^{*}$ & 515.7 & 336.0 & 33 & $.754^{*}$ \\
\hline 9 & 410.8 & -5.8 & 534.9 & $.742^{*}$ & 66.1 & 337.0 & 24 & $.619^{*}$ \\
\hline 10 & 328.0 & -6.9 & 47 & .60 & 18.2 & 276 & 9 & $.848^{*}$ \\
\hline 11 & 494.2 & -1.5 & 527.4 & .042 & 516.6 & 359.8 & 36 & $.360^{*}$ \\
\hline 12 & 473.7 & 3.2 & 405.9 & .090 & 433.0 & 718.0 & 36 & $.613^{*}$ \\
\hline 13 & 451.8 & -3.0 & 515.4 & $.313^{*}$ & 546.6 & 426.0 & 9 & .596 \\
\hline 14 & 390.7 & -1.6 & 425.8 & $.163^{*}$ & 495.3 & 382.6 & 3 & $.352^{\prime}$ \\
\hline 15 & 277.6 & 0.0 & 276.8 & .000 & 253.1 & 284.3 & 9 & $.200^{\prime}$ \\
\hline 16 & 475.1 & 0.1 & 473.2 & .000 & 379.0 & 482.5 & 3 & .149 \\
\hline$M$ & 408.3 & -2.5 & 461.3 & .254 & 449.6 & 378.3 & 19.8 & .459 \\
\hline$S D$ & 74.2 & 3.1 & 83.3 & .243 & 86.3 & 124.0 & 12.1 & .201 \\
\hline
\end{tabular}

Note-The mean column contains means of the 42 trial medians. Break is the last trial belonging to Step 1. VAF is the variance accounted for by the best-fitting function. *Denotes proportions of variance accounted for greater than .154 , the critical value of $r^{2}$ corresponding to statistical significance at the .01 level under the linear regression model with $40 \mathrm{df}$. 
Table 2

Interresponse Interval (IRI) and Semiinterquartile Range (SIQR) Regressed on Interstimulus Interval (ISI) by Subject

\begin{tabular}{crrrrrr}
\hline & & \multicolumn{3}{c}{ IRI } & \multicolumn{3}{c}{ SIQR } \\
\cline { 3 - 6 } \cline { 5 - 6 } Subject & Slope & Intercept & VAF $\left(r^{2}\right)$ & Slope & Intercept & VAF $\left(r^{2}\right)$ \\
\hline 1 & .977 & 6.014 & .995 & .024 & 1.306 & .806 \\
2 & .994 & -1.868 & .999 & .035 & -1.168 & .733 \\
3 & .972 & 24.208 & .994 & .041 & -4.094 & .900 \\
4 & .963 & -9.393 & .994 & .025 & 6.412 & .528 \\
5 & .937 & 16.337 & .996 & .034 & -1.335 & .920 \\
6 & .971 & 1.316 & .999 & .015 & 8.301 & .723 \\
7 & .961 & 17.233 & .998 & .029 & 0.867 & .883 \\
8 & 1.033 & -9.070 & .994 & .029 & 0.957 & .892 \\
9 & .961 & 10.778 & .995 & .028 & 2.391 & .773 \\
10 & .987 & 8.028 & .998 & .018 & 3.103 & .896 \\
11 & .924 & 24.800 & .992 & .032 & 2.360 & .748 \\
12 & .926 & 29.478 & .996 & .016 & 2.529 & .840 \\
13 & .940 & 15.095 & .995 & .021 & 3.620 & .655 \\
14 & .906 & 17.806 & .995 & .026 & -0.836 & .826 \\
15 & 1.091 & -30.864 & .996 & .033 & 0.489 & .847 \\
16 & .986 & -0.878 & .991 & .022 & 3.013 & .794 \\
$M$ & .971 & 8.613 & .995 & .027 & 1.745 & .798 \\
$S D$ & .043 & 14.443 & .002 & .007 & 2.905 & .101 \\
\hline
\end{tabular}

Note-VAF is the variance accounted for by the best-fitting function.

Within-category changes in preferred rate may be the rule, with only a few subjects shifting from one temporal category to another over sessions.

The trend over sessions influences our interpretation of the bimodal distribution of trials in Figure 3. The leftward component of the distribution is composed partly of trials from subjects whose preferred IRIs were short and remained so over sessions. These subjects showed small changes and seemed to operate entirely in a "fast" temporal category whose natural period was estimated to be $250 \mathrm{msec}$ by Collyer et al. (1992). The leftward component also contains trials from subjects who exhibited large, apparently between-category, changes, with their last few trials belonging to the fast category. The rightward component of the distribution is composed of trials from subjects who apparently selected preferred IRIs entirely within a "slower" temporal category whose natural period was estimated to be $513 \mathrm{msec}$ (Collyer et al., 1992). However, the bulk of this distribution lies to the left of $513 \mathrm{msec}$; one interpretation of this leftward shift is that, within a temporal category, there is a preference for faster over slower performance. Under this interpretation, the relative paucity of cases between the two modes of the distribution serves to locate the slow half of the fast category whose natural period is near $250 \mathrm{msec}$.

\section{Constrained Tapping Performance}

Percent residual bias. Each subject's five trials at each ISI were summarized by the mean of the five trial median IRIs and the mean of the five trial semiinterquartile ranges. Linear regression was used to measure overall change as a function of ISI. Slope and intercept estimates from these analyses are given in Table 2 .

Residual bias was defined as the difference between IRI and the value predicted from linear regression. PRB was defined as this difference, expressed as a percentage of the linear prediction. A subject's oscillator signature is PRB as a function of ISI.

Averaging of oscillator signatures. PRB values were averaged across the 16 subjects to obtain an average PRB signature. The smoothed values of each subject were also averaged to obtain an average smoothed signature. These two functions are shown in Figure 4. The average smoothed function accounted for $69 \%$ of the variance in the average PRB signature, which indicates that smoothing captures a great deal of the nonlinear structure remaining in PRB.

As a check that this nonlinear structure was not an artifact of smoothing itself, the averaging operations were also carried out on randomly scrambled versions of the 16 sets of data. A spreadsheet was used to randomly resort each subject's PRB values independently of ISI. The scrambled PRB data sets were then smoothed. The scrambled data sets and the smoothed versions were separately

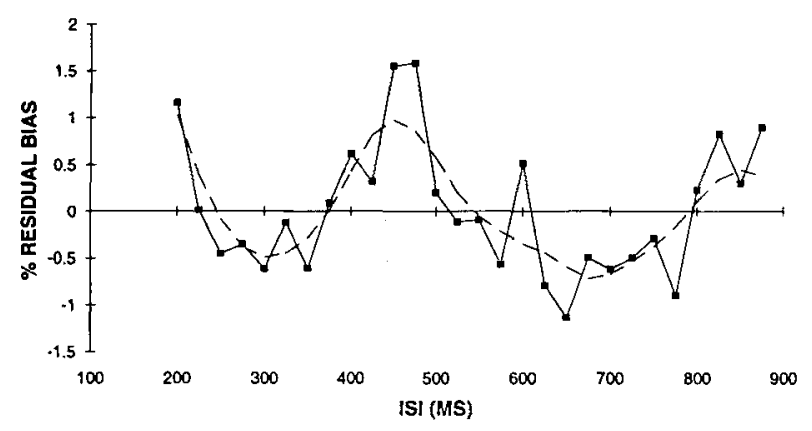

Figure 4. Averages of 16 oscillator signatures and the corresponding 16 smoothed signatures from the $\mathbf{4 2 5 3 H}$-twice filter. 
averaged. The average smoothed function in this case accounted for less than $1 \%$ of the variance in the average scrambled PRB function. Thus, concern that smoothing itself may create an appearance of nonlinear structure appears unwarranted.

The average smoothed function has negative-going zero crossings near 250 and $550 \mathrm{msec}$, and positive-going crossings near 375 and $800 \mathrm{msec}$. These values agree well with the average locations of zero crossings from the 5 subjects studied by Collyer et al. (1992). Revised estimates, based on both studies, of the negative-going zero crossings are 250 and $541 \mathrm{msec}$; revised positive-going zero crossings are 384 and $788 \mathrm{msec}$.

Within-subject reliability of oscillator signatures. Two replications of the constrained task, with slightly different ranges of ISI, were carried out by Subject 10 . The original PRB data from these replications are shown in Figure 5A, and the smoothed versions are shown in Figure $5 \mathrm{~B}$. The similar forms of the replications demonstrates the within-subject reliability of the oscillator signature and, by inference, the stability of a subject's timing mechanism. The two replications were carried out several months apart and with different random orders of administration of the ISI conditions.

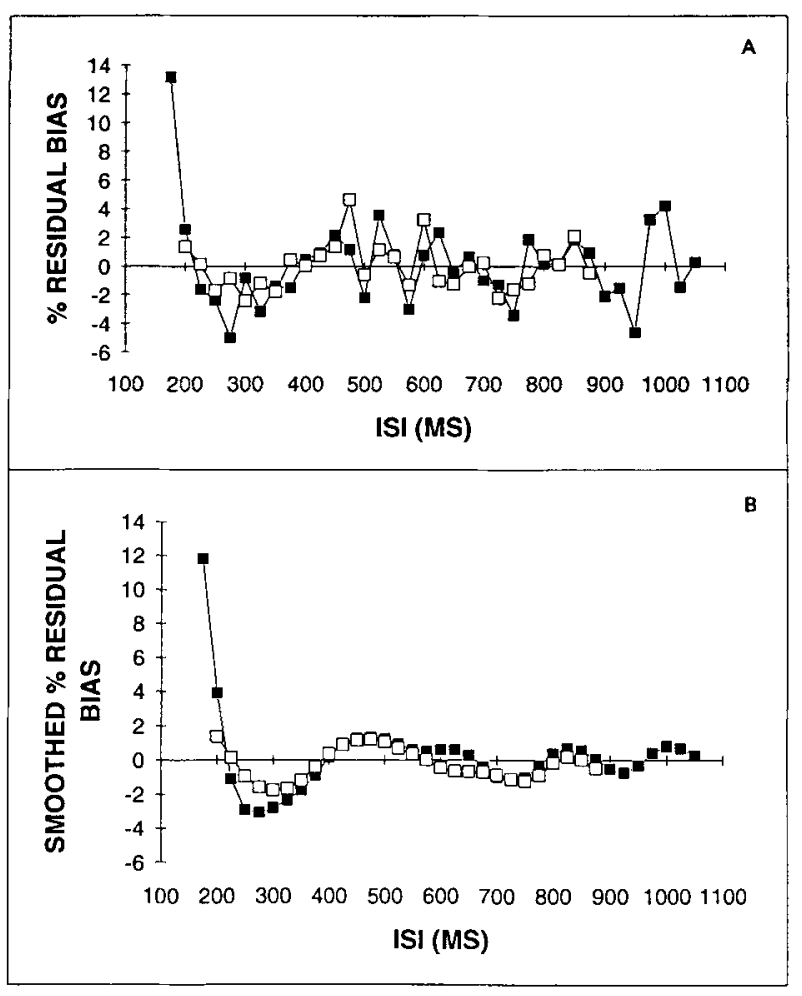

Figure 5. Within-subject replications of Subject 10's oscillator signature. (A) Superposition of PRB functions; (B) Superposition of smoothed functions. The replications were separated by about 6 months. Different random orders of interstimulus interval conditions were used.
Table 3

Descriptors of Individual Oscillator Signatures

\begin{tabular}{ccc}
\hline Subject & $\begin{array}{c}\text { Amplitude } \\
\text { (SD of smooth) }\end{array}$ & $\begin{array}{c}\text { Location } \\
\text { (Positive peak*) }\end{array}$ \\
\hline 1 & 0.83 & 475 \\
2 & 0.39 & 275 \\
3 & 1.33 & 450 \\
4 & 1.05 & 475 \\
5 & 1.55 & 450 \\
6 & 0.70 & 450 \\
7 & 1.60 & 475 \\
8 & 2.31 & 400 \\
9 & 1.23 & 425 \\
10 & 0.90 & 475 \\
11 & 1.85 & 600 \\
12 & 2.27 & 475 \\
13 & 1.74 & 450 \\
14 & 1.51 & 350 \\
15 & 3.01 & 525 \\
16 & 1.81 & 438 \\
$M$ & 1.51 & 449 \\
$S D$ & 0.67 & 71 \\
\hline
\end{tabular}

*In milliseconds.

Amplitude of oscillator signatures. The nonlinearity in a subject's performance could be more or less pronounced. A convenient measure of the amplitude of an individual oscillator signature is the standard deviation of the 28 smoothed PRB values. This measure, given for each subject in Table 3 , ranged from 0.39 to 3.01 , with a median of 1.53. The signatures of Subject 10, near the first quartile of amplitude (0.90), and Subject 11, near the third quartile (1.85), are shown in Figure 6. The figure shows the PRB and smoothed versions of the data, as well as confidence intervals at each ISI based on the null hypothesis that $\mathrm{PRB}=0$.

Location of oscillator signatures. The location of an oscillator signature can be indexed by the ISI associated with some feature of interest. Since individual oscillator signatures differ in form (for reasons not yet understood), it is not clear that there is one best marker of location; the relative locations of several individuals may depend to some degree on the feature chosen. The feature chosen for the present analysis was the peak positive bias of the smoothed signature.

For several subjects, the overall maximum positive bias was associated with ISI $=200 \mathrm{msec}$, the fastest tapping condition. Because positive bias might be due to motor difficulty at fast rates, we required that peak positive bias be defined as a local maximum of the smoothed signature, with smaller values on either side. In the averaged signature of Figure 4, the ISI associated with peak positive bias, defined in this way, was $450 \mathrm{msec}$. Individual values of peak positive bias, shown in Table 3, ranged from 275 to $600 \mathrm{msec}$, with a median of $450 \mathrm{msec}$. The signatures of Subject 9, near the first quartile (425), and Subject 7, near the third quartile (475), are shown in Figure 7.

Drift. Within-trial drift of IRI as a function of ISI was examined for each subject. The median difference between adjacent IRIs, and the semiinterquartile range of these differences, were averaged across the five trials at each ISI. 
Linear regression was used to measure the overall change in drift as a function of ISI. Drift tended to be negative and to become more pronounced at larger ISIs. The variability of drift also increased with ISI. Table 4 shows the slopes and intercepts of the regressions.

The nonlinear component of drift was examined to determine whether it might reflect the same nonlinearity measured by the oscillator signature. The residuals from the drift regression lines were calculated for each subject, smoothed, and correlated with the subject's smoothed oscillator signature. The 16 correlations ranged from -0.63 to +0.57 ; half were positive and half were negative, indicating that the nonlinear component of drift was not consistently related to the nonlinear component of IRI.

\section{Relationships Between Constrained and Unconstrained Performance}

To compare preferred IRIs to the oscillator signature, individual smoothed signatures were aligned at the subject's median preferred interval and then averaged. Figure 8 shows the resulting function. (Intervals with values from fewer than 10 subjects were truncated.) On average, the median preferred IRI was associated with distinctly positive bias. PRB associated with the preferred interval was positive for 13 of the 16 subjects (binomial probability $p=.0105$ ). Recall that the ISI of peak positive bias

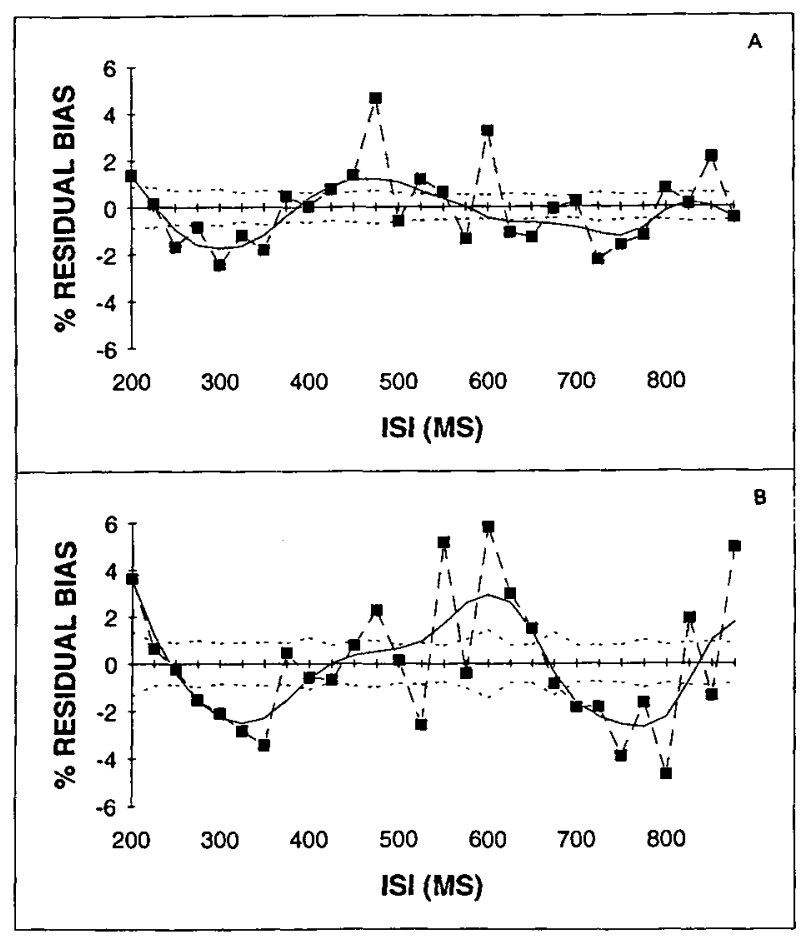

Figure 6. The amplitude of an oscillator signature was defined as the standard deviation of the 28 smoothed values. (A) Subject 10, amplitude $=0.90$, near the 1st quartile; (B) Subject 11, amplitude $=$ 1.85 , near the 3 rd quartile. Confidence limits defining the $99 \%$ interval around $P R B=0$, based on each subject's within-trial variability, are indicated by the dotted lines.

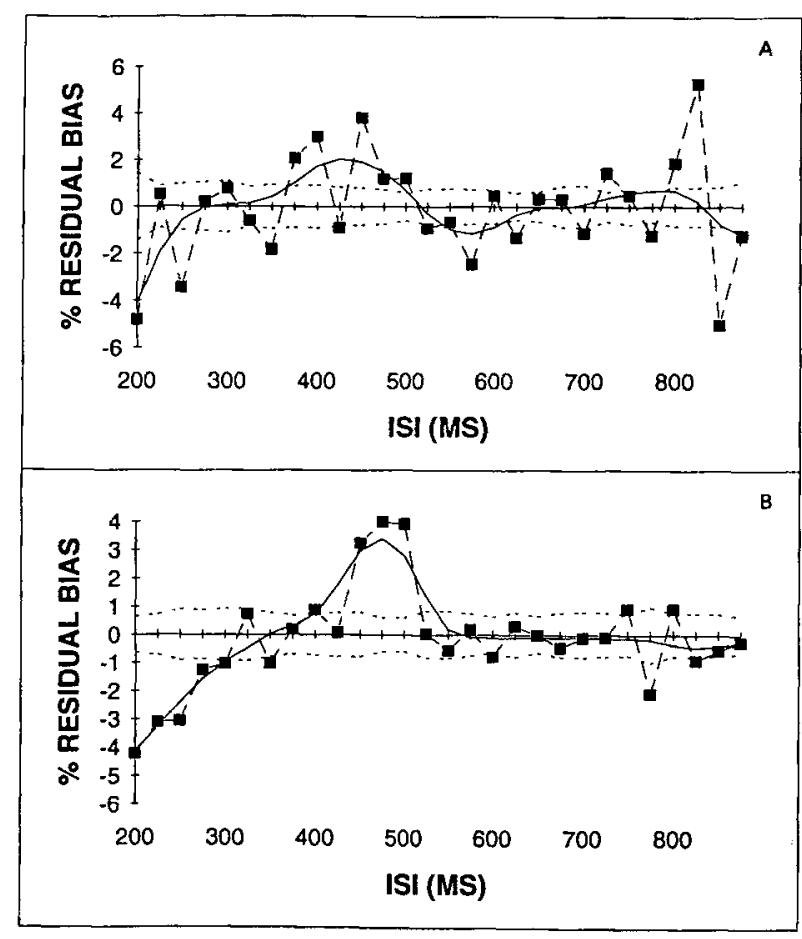

Figure 7. The location of a smoothed oscillator signature was indexed by the interstimulus interval associated with peak positive bias. (A) Subject 9, peak at $\mathbf{4 2 5}$ msec, near the 1st quartile; (B) Subject 7 , peak at 475 msec, near the 3rd quartile.

( $450 \mathrm{msec}$ ) was also the mode of the long-interval component in the bimodal distribution of preferred rates.

Table 5 shows the intercorrelations of two measures of constrained performance (amplitude and location of the oscillator signature) and three measures of unconstrained performance (overall median preferred IRI, and the Step 1 and Step 2 estimates from the step-model analysis of trend). The correlation between location (peak positive bias) and preferred rate, which was of special interest, was positive but did not attain significance.

\section{PROPOSED MODEL}

Any explanation of the oscillator signature should account for both the approximate equivalence of IRIs and ISIs at all interstimulus intervals between 200 and $875 \mathrm{msec}$, and the small systematic residual bias, with its multiple zero crossings in the range of 200 to $875 \mathrm{msec}$. It would also be desirable for an explanation of the oscillator signature to encompass the bimodal distribution of preferred IRIs.

Both single-oscillator models of timing (see, e.g., Gibbon, Church, \& Meck, 1984) and multiple-oscillator models (see, e.g., Church \& Broadbent, 1990, 1991) are sufficient to account for the linear relationship between the IR Is and the ISIs. But single-oscillator models of timing do not account for the systematic residuals. After identifying the 
Table 4

Linear Regression Analysis of Drift as a Function of ISI

\begin{tabular}{|c|c|c|c|c|c|c|}
\hline \multirow[b]{2}{*}{ Subject } & \multirow[b]{2}{*}{ Slope } & \multicolumn{2}{|c|}{ Drift } & \multicolumn{3}{|c|}{ SIQR } \\
\hline & & Intercept & $\operatorname{VAF}\left(r^{2}\right)$ & Slope & Intercept & $\operatorname{VAF}\left(r^{2}\right)$ \\
\hline 1 & -.003 & 0.462 & .232 & .031 & 4.665 & .610 \\
\hline 2 & -.005 & 1.224 & .206 & .044 & 0.469 & .619 \\
\hline 3 & -.002 & -0.361 & .059 & .044 & 0.609 & .854 \\
\hline 4 & -.001 & -0.903 & .014 & .030 & 13.661 & .322 \\
\hline 5 & -.003 & 0.153 & .176 & .041 & 1.949 & .896 \\
\hline 6 & -.003 & -0.400 & .123 & .012 & 17.758 & .470 \\
\hline 7 & -.001 & -0.233 & .032 & .034 & 4.650 & .819 \\
\hline 8 & .001 & -1.286 & .003 & .032 & 7.426 & .744 \\
\hline 9 & -.003 & -0.241 & .070 & .028 & 10.815 & .642 \\
\hline 10 & .000 & -0.207 & .000 & .021 & 7.040 & .794 \\
\hline 11 & -.002 & 0.026 & .026 & .040 & 8.322 & .429 \\
\hline 12 & -.004 & 1.480 & .239 & .023 & 4.557 & .778 \\
\hline 13 & -.002 & 0.252 & .083 & .016 & 12.225 & .310 \\
\hline 14 & -.003 & 0.586 & .138 & .032 & 1.821 & .712 \\
\hline 15 & -.008 & 2.215 & .406 & .045 & 4.006 & .796 \\
\hline 16 & -.002 & -0.409 & .057 & .026 & 7.518 & .662 \\
\hline$M$ & -.003 & 0.147 & .117 & .031 & 6.718 & .654 \\
\hline$S D$ & .002 & 0.893 & .111 & .010 & 4.930 & .184 \\
\hline
\end{tabular}

Note-Drift refers to change in IRI from one response to the next within a trial; units are in milliseconds per tap, and negative values denote speedup of tapping as a trial progresses. Thus, slope units are rather esoteric: milliseconds per tap per milliseconds of ISI. For example, the mean of -.003 indicates that an increase in ISI of $1,000 \mathrm{msec}$ would be associated with greater speedup, the change in drift being $3 \mathrm{msec}$ per tap. VAF is the variance accounted for by the best-fitting function.

oscillator signature result, we found that a multipleoscillator model also produced systematic residuals.

The multiple-oscillator, connectionist model of timing described by Church and Broadbent $(1990,1991)$ combines ideas about autoassociative memories (Anderson, Silverstein, Ritz, \& Jones, 1977) and about the representation of

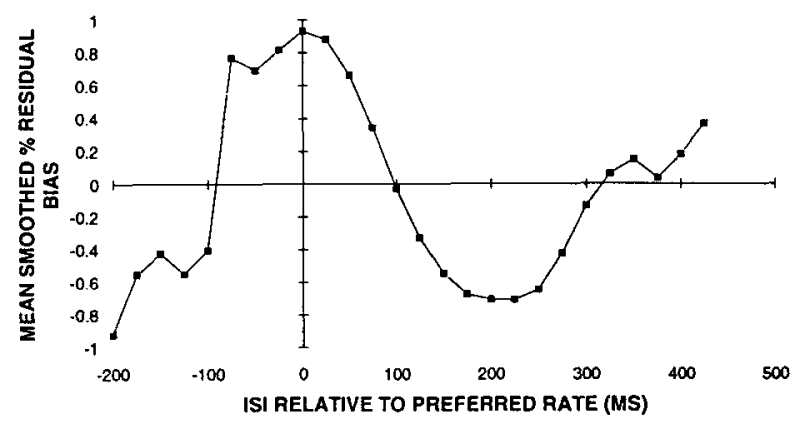

Figure 8. Average oscillator signature after alignment at the subject's preferred rate (denoted zero on the abscissa). The preferred interresponse interval is typically an interval that would be associated with positive bias in the constrained task.

Table 5

Intercorrelations of Constrained and Unconstrained Performance Measures

\begin{tabular}{lcccc}
\multicolumn{5}{c}{ Performance Measures } \\
\hline Location & Amplitude & Location & Preferred IRI & Step 1 \\
Preferred IRI & 0.42 & & & \\
Step 1 & 0.26 & 0.33 & & \\
Step 2 & -0.11 & 0.25 & $0.50^{*}$ & \\
\hline
\end{tabular}

$* p<.05$. time by multiple oscillators (Gallistel, 1990). An overview of the model for the storage of a time interval is as follows: A set of oscillators is postulated, each with a characteristic period and some variability. The half-phase of each oscillator is represented in a status indicator as a vector of $+1 \mathrm{~s}$ and $-1 \mathrm{~s}$. Working memory is represented as an autoassociation of this vector with itself (a matrix). During a training phase, reference memory is constructed by repeatedly combining the contents of the working-memory matrix representing a reinforced time interval with a reference-memory matrix.

An overview of the model for retrieval and decision is as follows: Another set of oscillators is postulated, each with a characteristic period and some variability. The halfphase of each oscillator is represented in a status indicator as a vector of $+1 \mathrm{~s}$ and $-1 \mathrm{~s}$. The product of the referencememory matrix and the status register provides an output vector. The cosine (normalized product) of the status indicator vector with the output vector provides a measure of similarity (between +1 and -1 ) of the representation of the current time to the remembered time. If the similarity is greater than some threshold value, a response will be made.

The simulations of Church \& Broadbent $(1990,1991)$ involved a timing system with 11 oscillators with periods of $200,400,800,1,600 \ldots$ msec. On each trial, the random variability was normally distributed with a mean of 0 and a standard deviation of .2 times the current period. Storage of new information into reference memory was a weighted average of $1 \%$ new information (working memory) with 99\% old information (reference memory). The threshold for responding was set at a similarity value of .5 . One thousand trials of training and 100 trials of testing were used. 
This simulation was used to generate three facts of temporal generalization in animals: (1) the mean response rate increases to a maximum near the time that food is typically available and then decreases; (2) on single trials, there is a period of low response rate followed by a period of high response rate that typically brackets the time that food is typically available, followed by another period of low response rate; and (3) the mean response rate is approximately the same function of relative time (time $\div$ time of food) for different times of food. Simulations were done with three stored times $(20,40$, and $80 \mathrm{sec})$.

The present simulation used the identical parameters although, for convenience, the base oscillator was set at 1 , with the series being $1,2,4,8$, and so forth, in arbitrary time units. Simulations were done with stored times from 20 to 80 , in intervals of 1 . Ten tests were made at each of these 61 intervals, and the median of the 10 tests was used in the figures. The mean IRI was approximately a linear function of the ISI (top panel of Figure 9), but the PRB function (bottom panel of Figure 9) had systematic deviations similar to those seen in empirical oscillator signatures. The magnitude of the bias was greater in the simulated than in the actual results, but adjustment of the variability parameter can reduce the magnitude of the bias.

Considering that the simulation used two oscillator periods (32 and 64) that were within the range of tested

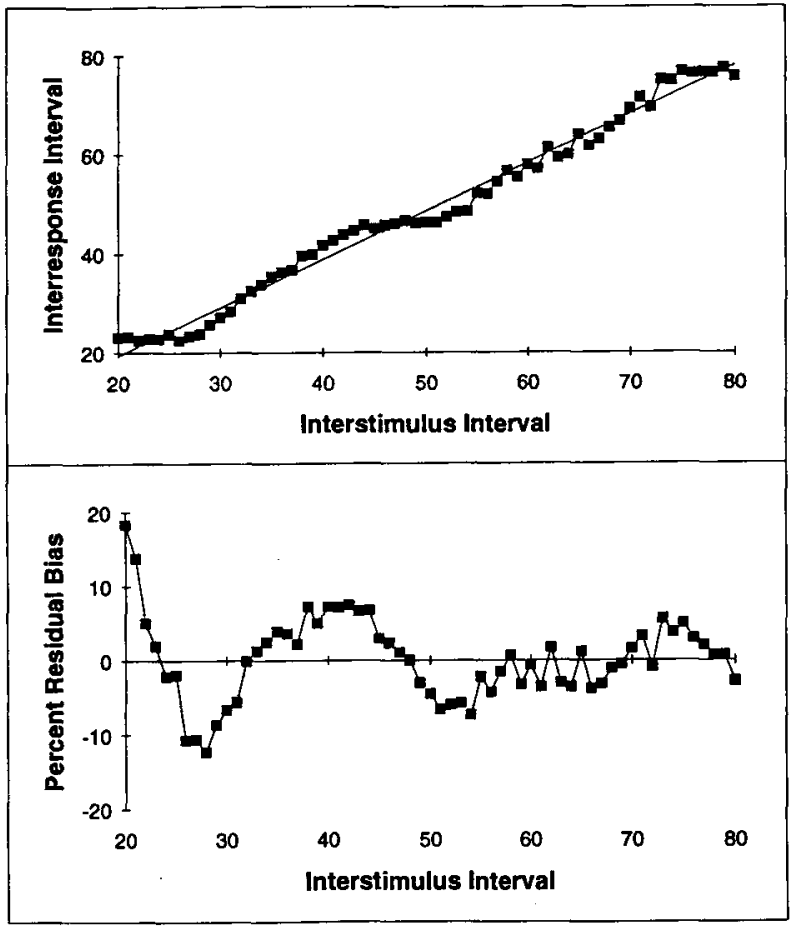

Figure 9. Simulation of constrained tapping data by the multipleoscillator connectionist model. Two of the oscillators had periods of 32 and 64 . The top panel shows that interresponse interval is an approximately linear function of interstimulus interval. The bottom panel shows that residuals from a best-fitting straight line exhibit an oscillator signature resembling the experimental result with human subjects.

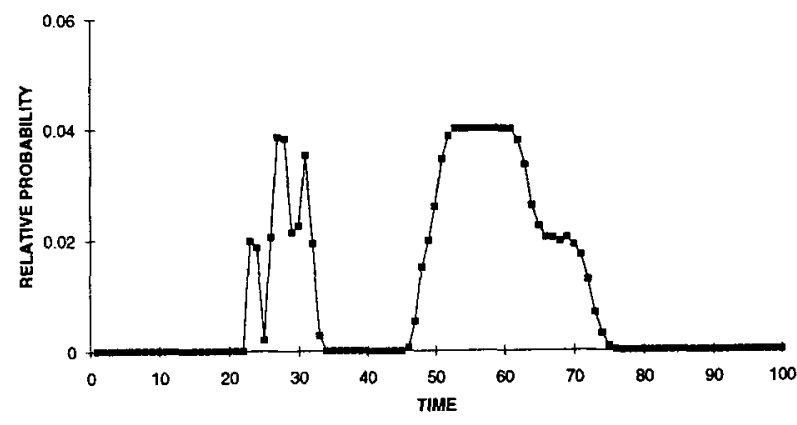

Figure 10. Simulation of unconstrained tapping data by the multiple-oscillator connectionist model. Two of the oscillators had periods of 32 and 64 . The bimodal distribution agrees qualitatively with the human experiment.

intervals, Figure 9 suggests that positive-going zero crossings are associated with oscillator periods in this model. Thus, if oscillator periods are considered as analogous to the lower-precision values of a rounding function, the simulated oscillator signature resembles a rounding-up rule more closely than a rounding-to-nearest rule.

The model was also used to simulate preferred-rate data (see Figure 10). During training, the model's reference memory was constructed to be "equipotential" for all times that were to be tested. That is, all values between 20 and 80 in steps of 1 were reinforced with equal probability for 100,000 training trials. During 1,000 trials of testing, the reference memory was probed with test times between 0 and 100 , and similarity values led to a response if they exceeded .5. On each trial, the random variability was normally distributed with a mean of 0 and a standard deviation of 0.02 . Figure 10 shows that the relationship between the tendency to respond and the test time was bimodal. The two modes were more distinct in the simulated than in the human results, but this feature could be adjusted by changing the variability parameter.

Each mode of the simulated data was located somewhat to the left of an oscillator value ( 32 or 64 ). Thus, although the number, spacing, and placement of the modes suggests that they are related to these two oscillators, the location of a mode would be a biased estimate of an oscillator period.

These simulation results are qualitative demonstrations of the model's possible value for understanding the timing mechanism. The conclusion is that a multiple-oscillator, connectionist model of timing can produce interresponse times that are approximately accurate at all interstimulus times but that have small, systematic residuals similar to those observed in constrained tapping, and can produce bimodally distributed preferred intervals.

\section{CONCLUSION}

Subjects in repetitive tapping tasks exhibit two phenomena that are difficult for single-oscillator theories of timing to explain. In constrained tapping, the function relating IRI to ISI contains a small nonlinearity, the oscillator signa- 
ture (Collyer et al., 1992), which was replicated here. In unconstrained tapping, subjects' preferred IR Is are distributed bimodally.

A multiple-oscillator view of timing provides alternative ways of conceptualizing several issues in both human and animal timing. One multiple-oscillator connectionist model (Church \& Broadbent, 1990, 1991) was shown to produce an oscillator signature and a bimodal distribution of unconstrained intervals. Many questions about the mechanism responsible for timing can be addressed in terms of this model and related multiple-oscillator models.

\section{REFERENCES}

Anderson, J. A., Silverstein, J. W., Ritz, S. A., \& Jones, R. S. (1977). Distinctive features, categorical perception, and probability learning: Some applications of a neural model. Psychological Review, 84, 413-451.

Church, R. M., \& Broadbent, H. A. (1990). Alternative representations of time, number, and rate. Cognition, 37, 55-81.

Church, R. M., \& Broadbent, H. A. (1991). A connectionist model of timing. In M. L. Commons, S. Grossberg, \& J. E. R. Staddon, Quantitative models of behavior: Neural networks and conditioning (pp. 225-240). Hillsdale, NJ: Erlbaum.

Collyer, C. E. (1985). Comparing strong and weak models by fitting them to computer-generated data. Perception \& Psychophysics, 38. 476-481.
Collyer, C. E., Broadbent, H. A., \& Church, R. M. (1990, June). Preferred rates of repetitive tapping are not strongly determined by physical dynamics. Paper presented at the American Psychological Society, Dallas.

Collyer, C. E., Broadbent, H. A., \& Church, R. M. (1992). Categorical time production: Evidence for discrete timing in motor control. Perception \& Psychophysics, 51, 134-144.

Galdistel, C. R. (1990). The organization of learning. Cambridge: MIT Press.

Gibbon, J., Church, R. M., \& Meck, W. H. (1984). Scalar timing in memory. In J. Gibbon \& L. G. Allan (Eds.), Timing and time perception (Annals of the New York Academy of Sciences, Vol. 423, pp. 5277). New York: New York Academy of Sciences.

MAKSIK, Y. (1991). Simpler, faster, more reliable photosensor circuits. Behavior Research Methods, Instruments, \& Computers, 23, 283-287.

TUKEY, J. W. (1977). Exploratory data analysis. Reading, MA: AddisonWesley.

Velleman, P. F., \& Hoaglin, D. C. (1981). Applications, basics, and computing of exploratory data analysis. Boston: Duxbury Press.

WING, A. M. (1980). The long and short of timing in response sequences. In G. E. Stelmach \& J. Requin (Eds.), Tutorials in motor behavior (pp. 469-486). Amsterdam: North-Holland.

WING, A. M., \& KRISTOFFERSon, A. B. (1973). The timing of interresponse intervals. Perception \& Psychophysics, 13, 455-460.

(Manuscript received October 12, 1992; revision accepted for publication September 14, 1993.) 